\title{
Efficient Hyperparameter Optimization under Multi-Source Covariate Shift
}

\author{
Masahiro Nomura ${ }^{*}$ \\ nomura_masahiro@cyberagent.co.jp \\ CyberAgent, Inc. \\ Tokyo, Japan
}

\author{
Yuta Saito* \\ saito@hanjuku-kaso.com \\ Hanjuku-kaso, Co., Ltd. \\ Tokyo, Japan
}

\begin{abstract}
A typical assumption in supervised machine learning is that the train (source) and test (target) datasets follow completely the same distribution. This assumption is, however, often violated in uncertain real-world applications, which motivates the study of learning under covariate shift. In this setting, the naive use of adaptive hyperparameter optimization methods such as Bayesian optimization does not work as desired since it does not address the distributional shift among different datasets. In this work, we consider a novel hyperparameter optimization problem under the multi-source covariate shift whose goal is to find the optimal hyperparameters for a target task of interest using only unlabeled data in a target task and labeled data in multiple source tasks. To conduct efficient hyperparameter optimization for the target task, it is essential to estimate the target objective using only the available information. To this end, we construct the variance reduced estimator that unbiasedly approximates the target objective with a desirable variance property. Building on the proposed estimator, we provide a general and tractable hyperparameter optimization procedure, which works preferably in our setting with a no-regret guarantee. The experiments demonstrate that the proposed framework broadens the applications of automated hyperparameter optimization.
\end{abstract}

\section{CCS CONCEPTS}

- Computing methodologies $\rightarrow$ Machine learning; • Information systems $\rightarrow$ Data mining.

ACM Reference Format:

Masahiro Nomura and Yuta Saito. 2021. Efficient Hyperparameter Optimization under Multi-Source Covariate Shift. In Proceedings of the 30th ACM International Conference on Information and Knowledge Management (CIKM '21), November 1-5, 2021, Virtual Event, QLD, Australia. ACM, New York, NY, USA, 10 pages. https://doi.org/10.1145/3459637.3482336

\section{INTRODUCTION}

Hyperparameter optimization (HPO) has been a pivotal part of machine learning (ML) and contributed to achieving a good performance in a wide range of tasks [11]. It is widely acknowledged that

${ }^{*}$ Both authors contributed equally to this research.

Permission to make digital or hard copies of all or part of this work for personal or classroom use is granted without fee provided that copies are not made or distributed for profit or commercial advantage and that copies bear this notice and the full citation on the first page. Copyrights for components of this work owned by others than ACM must be honored. Abstracting with credit is permitted. To copy otherwise, or republish, to post on servers or to redistribute to lists, requires prior specific permission and/or a fee. Request permissions from permissions@acm.org.

CIKM '21, November 1-5, 2021, Virtual Event, QLD, Australia

(C) 2021 Association for Computing Machinery.

ACM ISBN 978-1-4503-8446-9/21/11 . .\$15.00

https://doi.org/10.1145/3459637.3482336 the performance of deep neural networks depends greatly on the configuration of the hyperparameters $[9,15,21]$. HPO is formulated as a special case of a black-box function optimization problem, where the input is a set of hyperparameters, and the output is a validation score. Among the black-box optimization methods, adaptive algorithms, such as Bayesian optimization (BO) $[8,13,31]$ have shown superior empirical performance compared with traditional algorithms, such as grid search or random search [3, 39].

One critical, but often overlooked assumption in HPO is the availability of an accurate validation score. However, in reality, there are many cases where we cannot access the ground-truth validation score of the target task of interest. For example, in display advertising, predicting the effectiveness of each advertisement, i.e., click-through rates (CTR), is important for showing relevant advertisements (ads) to users. Therefore, it is necessary to conduct HPO before a new ad campaign starts. However, for new ads that have not yet been displayed to users, one cannot use labeled data to conduct HPO. Moreover, suppose you are a doctor who wants to optimize a medical plan for patients in a new, target hospital (hospital A) using ML. However, you have only historical medical outcome data from some source hospitals (hospitals B and C) different from the target one. In these situations, we have to tune hyperparameters that lead to a good ML model with respect to the target task without having its labeled data. These settings are considered as a generalization of covariate shift [32,36]. Covariate shift is a prevalent setting for supervised machine learning in the wild, where the feature distribution is different in the train (source) and test (target) tasks, but the conditional label distribution given the feature vector remains unchanged. In this case, the standard HPO procedure is infeasible, as one cannot utilize the labeled target task data and the true validation score of the ML model under consideration.

In this work, we extend the use of adaptive HPO algorithms to multi-source covariate shift (MS-CS). In this setting, we do not have labeled data for a target task (e.g., medical outcomes in a new hospital). However, we do have data for some source tasks following distributions that are different from the target task (e.g., historical outcomes in some other hospitals). The difficulty to conduct HPO under MS-CS is that the ground-truth validation score is inaccessible, as we cannot utilize the label information of the target task. It is thus essential to find a good estimator of the target objective, a performance of an ML model with respect to the target task, calculable with only available data.

A natural candidate approach is importance sampling [10, 36], which leads to an unbiased estimation by correcting the distributional shifts among different tasks. In our problem, however, a mere IS can lead to a sub-optimal conclusion, because the variance in the 
objective estimation can blow up due to the effect of some source tasks greatly different from the target task. To address this problematic variance issue, we first argue that the variance is the key to lead to an efficient HPO procedure in MS-CS. We then propose the variance reduced (VR) estimator, which achieves the optimal variance among a class of unbiased estimators by upweighting informative samples, i.e., samples of source tasks that are similar to the target task, based on a task divergence measure. We also show that our proposed estimator leads to a no-regret HPO even under MS-CS. Finally, empirical studies on synthetic and real-world datasets demonstrate that the proposed framework works properly compared to other possible heuristics.

Related Work. A typical HPO aims to find a better set of hyperparameters implicitly assuming that samples from the target task are available. As faster convergence is an essential performance metric of the HPO methods, the research community is moving on to the multi-source or transfer settings for which there are some previously solved related source tasks. By combining the additional source task information and the labeled target task dataset, one can improve the hyperparameter search efficiency, and thus reach a better solution with fewer evaluations [2, 6, 12, 24, 26-30, 34, 37, 42, 44]. A critical difference between multi-source HPOs and our MS-CS is the existence of labels for the target task. Previous studies usually assume that analysts can utilize the labeled target data. However, as discussed above, this is often unavailable, and thus, most of these methods are infeasible in practice.

Another related field is model evaluation under covariate shift, whose objective is to evaluate the performance of ML models with respect to the target task using only a relevant single source dataset $[36,45,46]$. These studies build on the importance sampling (IS) method [10,36] to obtain an unbiased estimate of ground-truth model performance. While our proposed methods are also based on IS, a major difference is that we assume that there are multiple source datasets following different distributions. We demonstrate that, in the multi-source setting, the previous IS method can fail and propose an estimator satisfying the optimal variance property. Moreover, as these methods are specific to model evaluation, the connection between the IS-based estimation techniques and automated HPO methods has not yet been explored despite their possible broad applications. Consequently, we are the first to theoretically show the no-regret guarantee of adaptive HPO under MS-CS and empirically evaluate its possible combination with the IS-based unbiased estimation.

Contributions. Our contributions are summarized as follows:

- We formulate a novel HPO setting under MS-CS.

- We construct the VR estimator, which achieves the optimal variance among a reasonable class of unbiased estimators.

- We show that our proposed estimator leads to a no-regret HPO even under MS-CS where labeled target task data are unavailable.

- We empirically demonstrate that the proposed procedure works favorably in MS-CS setting.

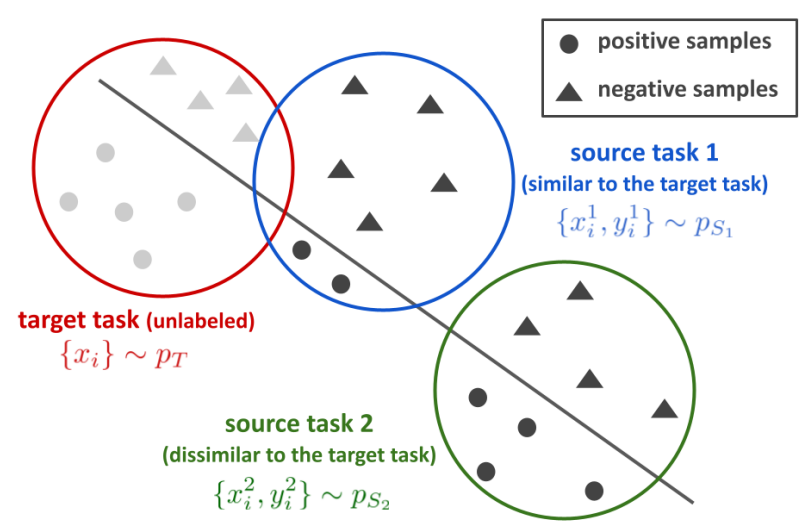

Figure 1: Multi-Source Covariate Shift (MS-CS)

Note: In MS-CS, we aim to find the best hyperparameters for the target task using unlabeled samples from the target task and labeled datasets from source tasks. Some source tasks are similar to the target (e.g., source task 1), while others are dissimilar (e.g., source task 2). Note that the decision boundary is the same for all tasks (c.f., Assumption 2.).

\section{SETUP}

Here, we formulate HPO under MS-CS. Let $\mathcal{X} \subseteq \mathbb{R}^{d}$ be the $d$ dimensional input space and $\mathcal{Y} \subseteq \mathbb{R}$ be the real-valued output space. We use $p_{T}(x, y)$ to denote the joint probability density function of the input and output variables of the target task. The objective of this work is to find the best set of hyperparameters $\theta$ with respect to the target distribution:

$$
\theta^{*} \in \underset{\theta \in \Theta}{\arg \min } f_{T}(\theta),
$$

where $\Theta$ is a pre-defined hyperparameter search space and $f_{T}(\theta)$ is the target task objective, which is defined as the generalization error over the target distribution:

$$
f_{T}(\theta):=\mathbb{E}_{(X, Y) \sim p_{T}}\left[L\left(h_{\theta}(X), Y\right)\right],
$$

where $L: \mathcal{Y} \times \mathcal{Y} \rightarrow \mathbb{R}_{\geq 0}$ is a bounded loss function such as the zero-one loss. $h_{\theta}: \mathcal{X} \rightarrow \mathcal{Y}$ is an arbitrary machine learning model defined by a set of hyperparameters $\theta \in \Theta$, which predicts the output values using the input vectors.

In a standard hyperparameter optimization setting $[4,11,33]$, labeled i.i.d. validation samples $\mathcal{D}_{T}^{\text {labeled }}:=\left\{x_{i}, y_{i}\right\}_{i=1}^{n_{T}} \sim p_{T}$ are available, and one can easily estimate the target objective in Eq. (2) by the following empirical mean:

$$
\hat{f}_{T}\left(\theta ; \mathcal{D}_{T}^{\text {labeled }}\right):=\frac{1}{n_{T}} \sum_{i=1}^{n_{T}} L\left(h_{\theta}\left(x_{i}\right), y_{i}\right) .
$$

A hyperparameter optimization is conducted directly minimizing Eq. (3) as a reasonable replacement for the ground-truth objective $f_{T}(\theta)$ in Eq. (2).

In contrast, under MS-CS, labels of the target task are unobservable; we can use only unlabeled target validation samples denoted as $\mathcal{D}_{T}:=\left\{x_{i}\right\}_{i=1}^{n_{T}}$. Instead, we can utilize multiple source task datasets, i.e., $\left\{\mathcal{D}_{S_{j}}\right\}_{j=1}^{k}$ where $j$ is a source task index and $k$ denotes the number of source tasks. Each source task is defined as i.i.d. labeled samples: $\mathcal{D}_{S_{j}}:=\left\{x_{i}^{j}, y_{i}^{j}\right\}_{i=1}^{n_{S_{j}}} \sim p_{S_{j}}$ where $p_{S_{j}}(x, y)$ is 
a joint probability density function that characterizes the source task $j$. Note here that the marginal input distributions of the target and source tasks are different, i.e., $p_{T}(x) \neq p_{S_{j}}(x), \forall j \in\{1, \ldots, k\}$. Finally, let we use $n:=\sum_{j=1}^{k} n_{S_{j}}$ to denote the total number of samples in the source tasks.

Regarding the target and source distributions, we make the following assumptions.

AsSUMPTION 1. Source tasks have support for the target task, i.e., $p_{T}(x)>0 \Rightarrow p_{S_{j}}(x)>0, \forall x \in \mathcal{X}, \forall j \in\{1, \ldots, k\}$.

ASSUMPTION 2. Conditional output distributions remain the same between the target and all the source tasks, i.e., $p_{T}(y \mid x)=p_{S_{j}}(y \mid x)$, $\forall j \in\{1, \ldots, k\}$.

The above assumptions are common in the covariate shift literature [32] and suggest that the input-output relation is the same, but the input distributions are different for the target and source task distributions. ${ }^{1}$

One critical difficulty of MS-CS is that the simple approximation by the empirical mean in Eq. (3) is infeasible, as the labeled target dataset is unavailable. It is thus essential to estimate the target task objective $f_{T}$ accurately using only an unlabeled target dataset and labeled multiple source datasets.

\section{METHOD}

In this section, we first define a class of unbiased estimators for the target objective by applying importance sampling to our setting. Any estimator within this class provides an unbiased target objective. However, optimizing some of them can be sub-optimal, as they can have a large variance when the target and source distributions differ greatly. To overcome the variance issue, we propose the variance reduced estimator, which achieves the minimum achievable variance among the class of unbiased estimators.

\subsection{A Class of Unbiased Estimators}

A natural method to approximate the target task objective under distributional shifts is to use importance sampling [32]. It addresses the distributional shifts among different tasks using the following density ratio.

DEFINITON 1. (Density Ratio) For any $(x, y) \in \mathcal{X} \times \boldsymbol{Y}$ with a positive source density $p_{S_{j}}(x, y)>0$, the density ratio between the target and the source task distributions is

$$
w_{S_{j}}(x, y):=\frac{p_{T}(x, y)}{p_{S_{j}}(x, y)}=\frac{p_{T}(x)}{p_{S_{j}}(x)}=w_{S_{j}}(x)
$$

where $w_{S_{j}}(x) \in[0, C]$ for a positive constant $C$. The equalities are derived from Assumption 2.

Using the density ratio, we define a class of unbiased estimators for the target task objective.

DEFINITON 2. ( $\boldsymbol{\lambda}$-unbiased Estimator) For a given set of hyperparameters $\theta \in \Theta$, the class of $\lambda$-unbiased estimators for the target task

\footnotetext{
${ }^{1}$ These assumptions seem to be strict, but in fact, they are relatively reasonable given that the general HPO literature implicitly assumes that the train-test distributions are completely the same, i.e., $p_{T}(x, y)=p_{S_{j}}(x, y), \forall j \in\{1, \ldots, k\}$.
}

objective is defined as

$$
\hat{f}_{\lambda}\left(\theta ;\left\{\mathcal{D}_{S^{j}}\right\}_{j=1}^{k}\right):=\sum_{j=1}^{k} \lambda_{j} \sum_{i=1}^{n_{S_{j}}} w_{S_{j}}\left(x_{i}^{j}\right) \cdot L\left(h_{\theta}\left(x_{i}^{j}\right), y_{i}^{j}\right)
$$

where $\lambda=\left\{\lambda_{1}, \ldots \lambda_{k}\right\}$ is a set of weights for source tasks that satisfies $\lambda_{j} \geq 0$ and $\sum_{j=1}^{k} \lambda_{j} n_{S_{j}}=1$ for all $j \in\{1, \ldots k\}$.

This estimator is a generalization of the importance weighted cross-validation [36] to the multi-source setting and is statistically unbiased for the ground-truth target task objective, i.e., for any given $\theta$ and $\lambda$, we have $\mathbb{E}\left[\hat{f}_{\lambda}\left(\theta ;\left\{\mathcal{D}_{S^{j}}\right\}_{j=1}^{k}\right)\right]=f_{T}(\theta)$. This is derived as follows.

$$
\begin{aligned}
\mathbb{E} & {\left[\hat{f}_{\lambda}\left(\theta ;\left\{\mathcal{D}_{S^{j}}\right\}_{j=1}^{k}\right)\right] } \\
& =\sum_{j=1}^{k} \lambda_{j} \sum_{i=1}^{n_{S^{j}}} \mathbb{E}_{(X, Y) \sim p_{S^{j}}}\left[w_{S^{j}}(X) \cdot L\left(h_{\theta}(X), Y\right)\right] \\
& =\sum_{j=1}^{k} \lambda_{j} \sum_{i=1}^{n_{S^{j}}} \mathbb{E}_{(X, Y) \sim p_{S^{j}}}\left[\frac{p_{T}(X, Y)}{p_{S^{j}}(X, Y)} \cdot L\left(h_{\theta}(X), Y\right)\right] \\
& =\sum_{j=1}^{k} \lambda_{j} \sum_{i=1}^{n_{S^{j}}} \mathbb{E}_{(X, Y) \sim p_{T}}\left[L\left(h_{\theta}(X), Y\right)\right] \\
& =\sum_{j=1}^{k} \lambda_{j} \sum_{i=1}^{n_{S^{j}}} f_{T}(\theta) \\
& =f_{T}(\theta) .
\end{aligned}
$$

Using an instance of the class of $\boldsymbol{\lambda}$-unbiased estimators is a valid approach for approximating the target task objective because of its unbiasedness. However, a question that comes up to our minds here is:

\section{What specific estimator should we use among the class of $\lambda$-unbiased estimators?}

A key property to answer this question is the variance of the estimators. For instance, let us define an immediate unbiased estimator $\hat{f}_{U B}\left(\theta ;\left\{\mathcal{D}_{S^{j}}\right\}_{j=1}^{k}\right):=n^{-1} \sum_{j=1}^{k} \sum_{i=1}^{n_{S_{j}}} w_{S_{j}}\left(x_{i}^{j}\right) L\left(h_{\theta}\left(x_{i}^{j}\right), y_{i}^{j}\right)$. This is a version of the $\lambda$-unbiased estimators with $\lambda_{j}=n_{S_{j}} / n$ for every $j$. We investigate the variance of this instance of the $\lambda$-unbiased estimators below.

PROPOSITION 1. (Variance of $\hat{f}_{U B}$ ) For a given set of hyperparame$\operatorname{ter} \theta \in \Theta$, the variance of $\hat{f}_{U B}$ is

$$
\begin{aligned}
& \mathbb{V}\left(\hat{f}_{U B}\left(\theta ;\left\{\mathcal{D}_{S^{j}}\right\}_{j=1}^{k}\right)\right) \\
& =\frac{1}{n^{2}} \sum_{j=1}^{k} n_{S^{j}}\left(\mathbb{E}_{(X, Y) \sim p_{S^{j}}}\left[w_{S^{j}}^{2}(X) \cdot L^{2}\left(h_{\theta}(X), Y\right)\right]-\left(f_{T}(\theta)\right)^{2}\right)
\end{aligned}
$$


Proof. Because the samples are independent, the variance can be represented as follows.

$$
\begin{aligned}
\mathbb{V}\left(\hat{f}_{U B}\left(\theta ;\left\{\mathcal{D}_{S^{j}}\right\}_{j=1}^{k}\right)\right) & =\frac{1}{n^{2}} \sum_{j=1}^{k} \sum_{i=1}^{n_{S^{j}}} \mathbb{V}\left(w_{S^{j}}(X) \cdot L\left(h_{\theta}(X), Y\right)\right) \\
& =\frac{1}{n^{2}} \sum_{j=1}^{k} n_{S^{j}} \cdot \mathbb{V}\left(w_{S^{j}}(X) \cdot L\left(h_{\theta}(X), Y\right)\right)
\end{aligned}
$$

where $\mathbb{V}\left(w_{S^{j}}(X) \cdot L\left(h_{\theta}(X), Y\right)\right)$ can be decomposed as

$$
\begin{aligned}
& \mathbb{V}\left(w_{S^{j}}(X) \cdot L\left(h_{\theta}(X), Y\right)\right)=\mathbb{E}_{(X, Y) \sim p_{S^{j}}}\left[w_{S^{j}}^{2}(X) \cdot L^{2}\left(h_{\theta}(X), Y\right)\right] \\
& \quad-\left(\mathbb{E}_{(X, Y) \sim p_{S^{j}}}\left[w_{S^{j}}(X) \cdot L\left(h_{\theta}(X), Y\right)\right]\right)^{2},
\end{aligned}
$$

From unbiasedness, $\mathbb{E}_{(X, Y) \sim p_{S^{j}}}\left[w_{S^{j}}(X) \cdot L\left(h_{\theta}(X), Y\right)\right]=f_{T}(\theta)$. Thus, we obtain Eq. (5).

The problem here is that, according to Proposition 1, its variance depends on the square value of the density ratio function, which can be large when there is a source task having a distribution that is dissimilar to that of the target task.

To illustrate this variance problem, we use a toy example given by $\left\{x_{1}, x_{2}\right\} \subseteq \mathcal{X},\left\{y_{1}, y_{2}\right\} \subseteq \mathcal{Y}, p\left(y_{1} \mid x_{1}\right)=p\left(y_{2} \mid x_{2}\right)=1, p\left(y_{2} \mid x_{1}\right)=$ $p\left(y_{1} \mid x_{2}\right)=0$. The loss values for possible tuples and the probability densities of the target and two source tasks are presented in Table 1. It shows that the target task $T$ is similar to the source task $S^{2}$, but its distribution is significantly different from that of $S^{1}$. For simplicity and without loss of generality, suppose there are two source task datasets given by $\mathcal{D}^{1}=\left\{\left(x_{1}^{1}, y_{1}^{1}\right)\right\}$ and $\mathcal{D}^{2}=\left\{\left(x_{1}^{2}, y_{1}^{2}\right)\right\}$. Then from Eq. (5), the variance of the unbiased estimator is about 64.27. Intuitively, this large variance is a result of the large variance samples from $S^{1}$. In fact, by dropping the samples of $S^{1}$ reduces the variance to 4.27 . From this example, we know that the unbiased estimator fails to make the most of the source tasks, and there is room to improve its variance by down-weighting the source tasks that are dissimilar to the target task.

Table 1: Dropping data samples from $S^{1}$ significantly lowers the variance of the unbiased estimator

\begin{tabular}{lcc}
\hline & $\left(x_{1}, y_{1}\right)$ & $\left(x_{2}, y_{2}\right)$ \\
\hline loss function: $L\left(h_{\theta}(x), y\right)$ & 10 & 1 \\
\hline target task $(T)$ distribution: $p_{T}(x, y)$ & 0.8 & 0.2 \\
source task $\left(S^{1}\right)$ distribution: $p_{S^{1}}(x, y)$ & 0.2 & 0.8 \\
source task $\left(S^{2}\right)$ distribution: $p_{S^{2}}(x, y)$ & 0.9 & 0.1 \\
\hline
\end{tabular}

\subsection{Variance Reduced Objective Estimator}

To deal with the potential large variance of the $\lambda$-unbiased estimators, we consider re-weighting samples from various source tasks based on their similarity to the target task. This builds on the idea that samples from source tasks close to the target task are much more informative in HPO than samples from dissimilar source tasks.
To lead to a more reasonable estimator, we first define a task divergence measure, which quantifies the similarity between the two tasks below.

DEFINITON 3. (Task Divergence Measure) The divergence between a source task distribution $p_{S_{j}}$ and the target task distribution $p_{T}$ is defined as

$$
\operatorname{Div}\left(T \| S^{j}\right):=\mathbb{E}_{p_{S_{j}}}\left[w_{S_{j}}^{2}(X) \cdot L^{2}\left(h_{\theta}(X), Y\right)\right]-\left(f_{T}(\theta)\right)^{2}
$$

This task divergence measure is large when the corresponding source distribution deviates significantly from the target task distribution. Building on this measure, we define the following estimator of the target task objective.

DEFINITON 4. (Variance Reduced Estimator) For a given set of hyperparameters $\theta \in \Theta$, the variance reduced estimator of the target task objective function is defined as

$$
\hat{f}_{V R}\left(\theta ;\left\{\mathcal{D}_{S^{j}}\right\}_{j=1}^{k}\right)=\sum_{j=1}^{k} \lambda_{j}^{\star} \sum_{i=1}^{n_{S^{j}}} w\left(x_{i}^{j}\right) \cdot L\left(h_{\theta}\left(x_{i}^{j}\right), y_{i}^{j}\right)
$$

where VR stands for variance reduced, $\mathcal{D}_{S^{j}}$ is any sample size $n_{S^{j}}$ of the i.i.d. samples from source task $j$, and $w_{S^{j}}(\cdot)$ is the true density ratio. $\lambda_{j}^{\star}$ is a weight for source task $j$, which is defined as

$$
\lambda_{j}^{\star}=\left(\operatorname{Div}\left(T \| S^{j}\right) \sum_{j=1}^{k} \frac{n_{S^{j}}}{\operatorname{Div}\left(T \| S^{j}\right)}\right)^{-1}
$$

Note that, for all $j \in\{1, \ldots k\}, \lambda_{j}^{\star} \geq 0$ and $\sum_{j=1}^{k} \lambda_{j}^{\star} n_{S^{j}}=1$.

The variance reduced estimator in Eq. (7) is statistically unbiased for the ground-truth target task objective in Eq. (2), i.e., for any given $\theta, \mathbb{E}\left[\hat{f}_{V R}\left(\theta ;\left\{\mathcal{D}_{S^{j}}\right\}_{j=1}^{k}\right)\right]=f_{T}(\theta)$, as it is an instance of the $\lambda$-unbiased estimators.

Then, we demonstrate that the variance reduced estimator in Eq. (7) is optimal in the sense that any other convex combination of a set of weights $\lambda=\left\{\lambda_{1}, \ldots \lambda_{k}\right\}$ that satisfies unbiasedness cannot provide a smaller variance.

THEOREM 2. (Variance Optimality; Extension of Theorem 6.4 of [1]) For any given set of weights $\lambda=\left\{\lambda_{1}, \ldots \lambda_{k}\right\}$ that satisfies $\lambda_{j} \geq 0$ and $\sum_{j=1}^{k} \lambda_{j} n_{S^{j}}=1$ for all $j \in\{1, \ldots k\}$, the following inequality holds

$$
\mathbb{V}\left(\hat{f}_{V R}\left(\theta ;\left\{\mathcal{D}_{S^{j}}\right\}_{j=1}^{k}\right)\right) \leq \mathbb{V}\left(\hat{f}_{\lambda}\left(\theta ;\left\{\mathcal{D}_{S^{j}}\right\}_{j=1}^{k}\right)\right)
$$

where $\hat{f}_{\lambda}\left(\theta ;\left\{\mathcal{D}_{S^{j}}\right\}_{j=1}^{k}\right)=\sum_{j=1}^{k} \lambda_{j} \sum_{i=1}^{n_{S^{j}}} w\left(x_{i}^{j}\right) \cdot L\left(h_{\theta}\left(x_{i}^{j}\right), y_{i}^{j}\right)$.

Proof. By following the same logic flow as in Proposition 1, the variance of the $\lambda$-unbiased estimator in Eq. (4) is

$$
\begin{aligned}
& \mathbb{V}\left(\hat{f}_{\lambda}\left(\theta ;\left\{\mathcal{D}_{S^{j}}\right\}_{j=1}^{k}\right)\right) \\
& =\sum_{j=1}^{k} \lambda_{j}^{2} n_{S^{j}}\left(\mathbb{E}_{(X, Y) \sim p_{S^{j}}}\left[w_{S^{j}}^{2}(X) \cdot L^{2}\left(h_{\theta}(X), Y\right)\right]-\left(f_{T}(\theta)\right)^{2}\right) \\
& =\sum_{j=1}^{k} \lambda_{j}^{2} n_{S^{j}} \cdot \operatorname{Div}\left(T \| S^{j}\right)
\end{aligned}
$$


Thus, by replacing $\lambda_{j}$ for $\left(\operatorname{Div}\left(T \| S^{j}\right) \sum_{j=1}^{k} \frac{n_{S^{j}}}{\operatorname{Div}\left(T \| S^{j}\right)}\right)^{-1}$, we have

$$
\begin{aligned}
\mathbb{V}\left(\hat{f}_{\lambda}\left(\theta ;\left\{\mathcal{D}_{S^{j}}\right\}_{j=1}^{k}\right)\right) & =\sum_{j=1}^{k}\left(\sum_{j=1}^{k} \frac{n_{S^{j}}}{\operatorname{Div}\left(T \| S^{j}\right)}\right)^{-2} n_{S^{j}} \cdot \operatorname{Div}\left(T \| S^{j}\right) \\
& =\sum_{j=1}^{k} \frac{n_{S^{j}} \operatorname{Div}\left(T \| S^{j}\right)}{\left(\operatorname{Div}\left(T \| S^{j}\right)\right)^{2}\left(\sum_{j=1}^{k} \frac{n_{S^{j}}}{\operatorname{Div}\left(T \| S^{j}\right)}\right)^{2}} \\
& =\left(\sum_{j=1}^{k} \frac{n_{S^{j}}}{\operatorname{Div}\left(T \| S^{j}\right)}\right)\left(\sum_{j=1}^{k} \frac{n_{S^{j}}}{\operatorname{Div}\left(T \| S^{j}\right)}\right)^{-2} \\
& =\left(\sum_{j=1}^{k} \frac{n_{S^{j}}}{\operatorname{Div}\left(T \| S^{j}\right)}\right)^{-1}
\end{aligned}
$$

Then, for any set of weights $\lambda=\left\{\lambda_{1}, \ldots \lambda_{k}\right\}$, we obtain the following variance optimality using the Cauchy-Schwarz inequality.

$$
\begin{aligned}
& \left(\sum_{j=1}^{k} \lambda_{j^{2}}^{2} n_{S^{j}} \cdot \operatorname{Div}\left(T \| S^{j}\right)\right)\left(\sum_{j=1}^{k} \frac{n_{S^{j}}}{\operatorname{Div}\left(T \| S^{j}\right)}\right) \geq\left(\sum_{j=1}^{k} \lambda_{j} n_{S^{j}}\right)^{2}=1 \\
& \Longrightarrow\left(\sum_{j=1}^{k} \lambda_{j}^{2} n_{S^{j}} \cdot \operatorname{Div}\left(T \| S^{j}\right)\right) \geq\left(\sum_{j=1}^{k} \frac{n_{S^{j}}}{\operatorname{Div}\left(T \| S^{j}\right)}\right)^{-1} \\
& \Longrightarrow \mathbb{V}\left(\hat{f}_{\lambda}\left(\theta ;\left\{\mathcal{D}_{S^{j}}\right\}_{j=1}^{k}\right)\right) \geq \mathbb{V}\left(\hat{f}_{V R}\left(\theta ;\left\{\mathcal{D}_{S^{j}}\right\}_{j=1}^{k}\right)\right)
\end{aligned}
$$

Theorem 2 suggests that the variance reduced estimator achieves a desirable variance property by weighting each source task based on its divergence from the target task.

Let us now return to the toy example in Table $1 .^{2}$ The values of the divergence measure for $S^{1}$ and $S^{2}$ are $\operatorname{Div}\left(T \| S^{1}\right)=252.81$ and $\operatorname{Div}\left(T \| S^{2}\right)=4.27$, respectively. This leads to the weights of $\lambda_{1}^{\star} \approx 0.017$ and $\lambda_{2}^{\star} \approx 0.983$. Then, we have 4.21 (variance of $\hat{f}_{V R}$ ) > 4.27 (variance when $S^{1}$ is dropped) > 64.27 (variance of $\hat{f}_{U B}$ ). It is now obvious that the variance reduced estimator $\hat{f}_{V R}$ performs much better than the unbiased estimator by optimally weighting all available source tasks.

\subsection{Hyperparameter Optimization Procedure}

We describe several detailed components of the proposed HPO procedure for the MS-CS setting.

Density Ratio Estimation: In general, the density ratio functions between the target and source tasks are unavailable and thus should be estimated beforehand. To estimate the density ratio, we employ unconstrained Least-Squares Importance Fitting ( $u$ LSIF) $[16,43]$, which minimizes the following squared error for the true density ratio function:

$$
\begin{aligned}
\hat{s} & =\underset{s \in \mathcal{S}}{\arg \min } \mathbb{E}_{p_{S^{j}}}\left[(w(X)-s(X))^{2}\right] \\
& =\underset{s \in \mathcal{S}}{\arg \min }\left[\frac{1}{2} \mathbb{E}_{p_{S^{j}}}\left[s^{2}(X)\right]-\mathbb{E}_{p_{T}}[s(X)]\right]
\end{aligned}
$$

\footnotetext{
${ }^{2}$ We refer to [1] to create the toy example.
}

where $\mathcal{S}$ is a class of measurable functions. Note that the empirical version of Eq. (8) is calculable with only unlabeled target and source task datasets.

Task Divergence Estimation: To utilize the variance reduced estimator, the task divergence measure Div $\left(T \| S^{j}\right)$ in Eq. (6) needs to be estimated from the available data. This can be done using the following empirical mean.

$$
\begin{aligned}
\widehat{\operatorname{Div}}\left(T \| S^{j}\right)= & \frac{1}{n_{S^{j}}} \sum_{i=1}^{n_{S^{j}}}\left(w\left(x_{i}^{j}\right) \cdot L\left(h_{\theta}\left(x_{i}^{j}\right), y_{i}^{j}\right)\right)^{2} \\
& -\left(\frac{1}{n_{S^{j}}} \sum_{i=1}^{n_{S^{j}}} w\left(x_{i}^{j}\right) \cdot L\left(h_{\theta}\left(x_{i}^{j}\right), y_{i}^{j}\right)\right)^{2}
\end{aligned}
$$

Data Splitting: To conduct HPO in our setting, we split the dataset of each source task $\mathcal{D}_{S^{j}}$ into three folds $\mathcal{D}_{S^{j}}^{\text {density }}, \mathcal{D}_{S^{j}}^{\text {train }}$, and $\mathcal{D}_{S^{j}}^{\text {val }}$ where each corresponds to the data for the density ratio estimation, training $h_{\theta}$, and evaluating $h_{\theta}$, respectively. First, we use $\mathcal{D}_{S^{j}}^{\text {density }}$ and $\mathcal{D}_{T}$ to estimate the density ratio function between the source task $j$ and the target task. Second, we use $\mathcal{D}_{S^{j}}^{\text {train }}$ to train $h_{\theta}$ for a given hyperparameter $\theta$ in an HPO procedure. Finally, we use $\mathcal{D}_{S^{j}}^{v a l}$ and $\mathcal{D}_{T}$ to estimate the target task objective and evaluate the performance of $\theta$. The data splitting is needed to ensure the variance optimality of our estimator.

Overall Procedure: Building on the above details, Algorithm 1 summarizes the high-level hyperparameter optimization procedure, which we develop for the MS-CS setting. In addition, we describe the specific hyperparameter optimization procedure when $\mathrm{BO}$ is used as OPT in Algorithm 2.

\section{REGRET ANALYSIS}

In this section, we analyze the regret bound under the MS-CS setting and prove that our proposed estimator achieves no-regret HPO. Here, we define a regret as

$$
r_{B}^{n}=f\left(\hat{\theta}_{B}^{*}\right)-f\left(\theta^{*}\right),
$$

where $f: \Theta \rightarrow \mathbb{R}$ is the ground-truth target task objective, $n=$ $\sum_{j=1}^{k} n_{S^{j}}$ is the total sample size among source tasks, and $B$ is the total number of evaluations. We also let $\theta^{*} \in \arg \min _{\theta \in \Theta} f(\theta)$, and $\hat{\theta}_{B}^{*} \in \arg \min _{\theta \in\left\{\theta_{1}, \cdots, \theta_{B}\right\}} \hat{f}_{n}(\theta)$ where $\hat{f}_{n}: \Theta \rightarrow \mathbb{R}$ is a target task objective estimated by the $\lambda$-unbiased estimator. Note that each of $\left\{\theta_{1}, \cdots, \theta_{B}\right\}$ is the hyperparameter selected among $B$ evaluations in the optimization.

To derive a regret bound, we first decompose the regret into the following three terms:

$$
\begin{aligned}
r_{B}^{n} & =f\left(\hat{\theta}_{B}^{*}\right)-f\left(\theta^{*}\right) \\
& =\left(f\left(\hat{\theta}_{B}^{*}\right)-\hat{f}_{n}\left(\hat{\theta}_{B}^{*}\right)\right)+\hat{f}_{n}\left(\hat{\theta}_{B}^{*}\right)+\left(\hat{f}_{n}\left(\hat{\theta}^{*}\right)-f\left(\theta^{*}\right)\right)-\hat{f}_{n}\left(\hat{\theta}^{*}\right) \\
& =\underbrace{\left(\hat{f}_{n}\left(\hat{\theta}_{B}^{*}\right)-\hat{f}_{n}\left(\hat{\theta}^{*}\right)\right)}_{(A)}+\underbrace{\left(f\left(\hat{\theta}_{B}^{*}\right)-\hat{f}_{n}\left(\hat{\theta}_{B}^{*}\right)\right)}_{(B)}+\underbrace{\left(\hat{f}_{n}\left(\hat{\theta}^{*}\right)-f\left(\theta^{*}\right)\right)}_{(C)},
\end{aligned}
$$

where $\hat{\theta}^{*} \in \arg \min _{\theta \in \Theta} \hat{f}_{n}(\theta)$. 

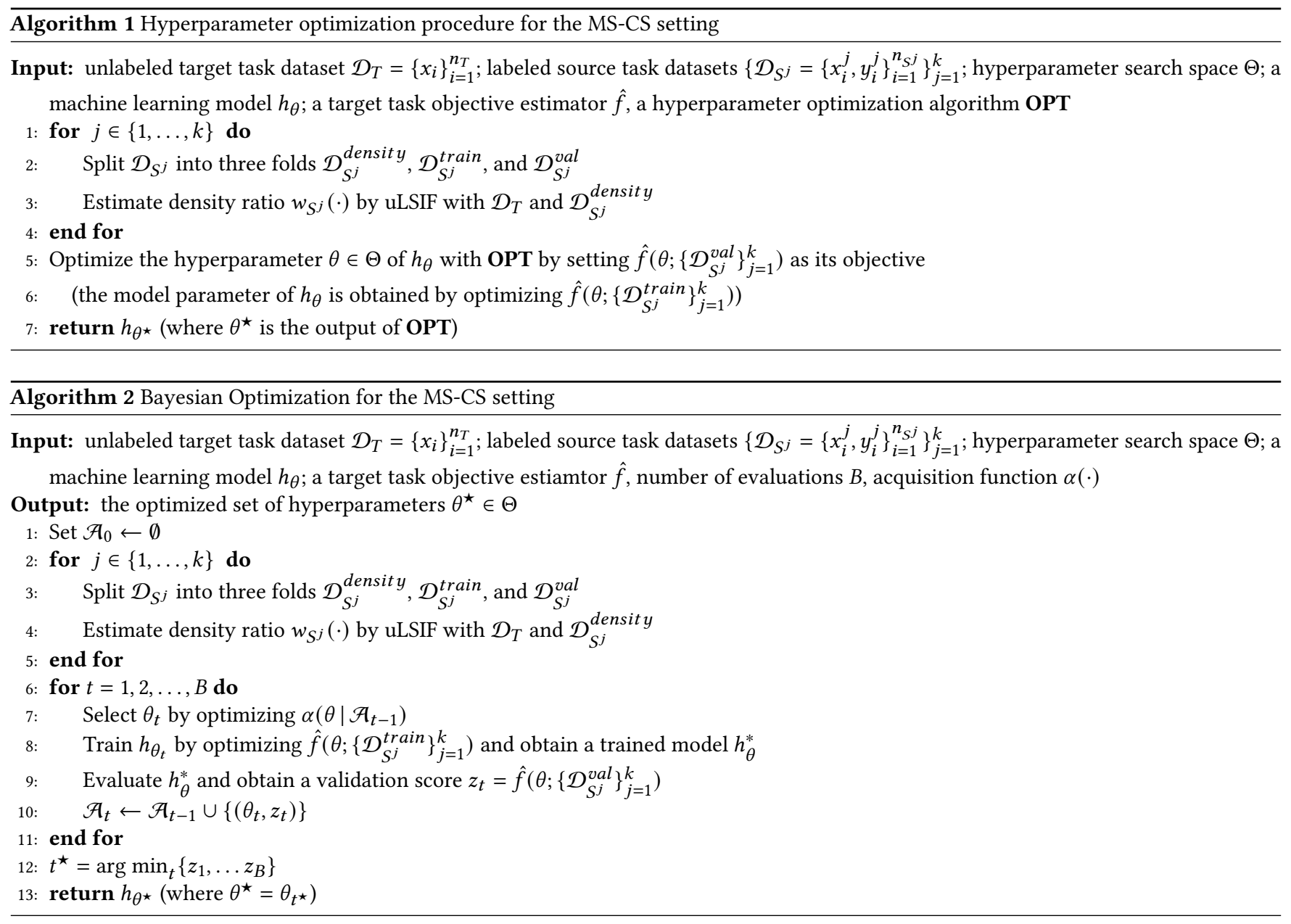

The term (A) is the simple regret obtained by optimizing the estimated target task objective $\hat{f}_{n}$. The term (B) is the difference between the true objective $f$ and the estimated objective $\hat{f}_{n}$ at $\hat{\theta}_{B}^{*}$, which is the solution of $\hat{f}_{n}(\theta)$. The term (C) is the difference between the minimum value of the estimated objective $\hat{f}_{n}$ and that of the true objective $f$. This decomposition is essential because we can bound the overall regret by finding a bound for each term.

We first state two lemmas, which will be used to bound the regret.

LEMMA 3. The following inequality holds with a probability of at least $1-\delta(\delta \in(0,1))$,

$$
\left(f\left(\hat{\theta}_{B}^{*}\right)-\hat{f}_{n}\left(\hat{\theta}_{B}^{*}\right)\right) \leq \sqrt{\mathbb{V}\left(\hat{f}_{n}\left(\hat{\theta}_{B}^{*}\right)\right) / \delta}
$$

Proof. By Chebyshev's inequality, we have

$$
\begin{aligned}
\mathbb{P}\left\{f\left(\hat{\theta}_{B}^{*}\right)-\hat{f}_{n}\left(\hat{\theta}_{B}^{*}\right) \geq c\right\} & \leq \mathbb{P}\left\{\left|f\left(\hat{\theta}_{B}^{*}\right)-\hat{f}_{n}\left(\hat{\theta}_{B}^{*}\right)\right| \geq c\right\} \\
& \leq \mathbb{V}\left(\hat{f}_{n}\left(\hat{\theta}_{B}^{*}\right)\right) / c^{2} .
\end{aligned}
$$

Putting the RHS as $\delta$ and solving it for $c$ completes the proof.
LEMMA 4. The following inequality holds with a probability of at least $1-\delta(\delta \in(0,1))$,

$$
\hat{f}_{n}\left(\hat{\theta}^{*}\right)-f\left(\theta^{*}\right) \leq \sqrt{\left(\mathbb{V}\left(\hat{f}_{n}\left(\theta^{*}\right)\right)+\mathbb{V}\left(\hat{f}_{n}\left(\hat{\theta}^{*}\right)\right)\right) / \delta} .
$$

Proof. By Chebyshev's inequality, we have

$$
\begin{aligned}
& \mathbb{P}\left\{\hat{f}_{n}\left(\hat{\theta}^{*}\right)-f\left(\theta^{*}\right) \geq c\right\} \\
& \leq \mathbb{P}\left\{\left|\hat{f}_{n}\left(\hat{\theta}^{*}\right)-f\left(\theta^{*}\right)\right| \geq c\right\} \\
& \leq \mathbb{P}\left\{\left|\hat{f}_{n}\left(\theta^{*}\right)-f\left(\theta^{*}\right)\right| \geq c \cup\left|\hat{f}_{n}\left(\hat{\theta}^{*}\right)-f\left(\hat{\theta}^{*}\right)\right| \geq c\right\} \\
& \leq \mathbb{P}\left\{\left|\hat{f}_{n}\left(\theta^{*}\right)-f\left(\theta^{*}\right)\right| \geq c\right\}+\mathbb{P}\left\{\left|\hat{f}_{n}\left(\hat{\theta}^{*}\right)-f\left(\hat{\theta}^{*}\right)\right| \geq c\right\} \\
& \leq \frac{1}{c^{2}}\left(\mathbb{V}\left(\hat{f}_{n}\left(\theta^{*}\right)\right)+\mathbb{V}\left(\hat{f}_{n}\left(\hat{\theta}^{*}\right)\right)\right) .
\end{aligned}
$$

Putting the RHS as $\delta$ and solving it for $c$ completes the proof.

Then, we state the main theorem.

THEOREM 5. (Regret Bound for MS-CS) When the $\lambda$-unbiased estimator with an arbitrary set of weights $\lambda$ is used as $\hat{f}\left(\theta ;\left\{\mathcal{D}_{S^{j}}^{v a l}\right\}_{j=1}^{k}\right)$, the following regret bound holds with a probability of at least $1-\delta(\delta \in$ 
$(0,1))$

$$
r_{B}^{n} \leq R_{n}+\sqrt{2 \mathbb{V}\left(\hat{f}_{n}\left(\hat{\theta}_{B}^{*}\right)\right) / \delta}+\sqrt{2\left(\mathbb{V}\left(\hat{f}_{n}\left(\theta^{*}\right)\right)+\mathbb{V}\left(\hat{f}_{n}\left(\hat{\theta}^{*}\right)\right)\right) / \delta}
$$

where $R_{n}=\hat{f}_{n}\left(\hat{\theta}_{B}^{*}\right)-\hat{f}_{n}\left(\hat{\theta}^{*}\right)$.

Proof. Putting Lemma 3 to the term (B) in Eq. (10) and Lemma 4 to the term (C) in Eq. (10) complete the proof.

When $\hat{f}_{n}$ is the proposed variance reduced estimator, $\mathbb{V}\left(\hat{f}_{n}(\cdot)\right)=$ $o(1)$. This means that the second and third terms in Eq. (11) are no-regret [35] with respect to $n$, i.e.,

$$
\left.\lim _{n \rightarrow \infty} \sqrt{2 \mathbb{V}\left(\hat{f}_{n}\left(\hat{\theta}_{B}^{*}\right)\right) / \delta}+\sqrt{2\left(\mathbb{V}\left(\hat{f}_{n}\left(\theta^{*}\right)+\mathbb{V}\left(\hat{f}_{n}\left(\hat{\theta}^{*}\right)\right)\right) / \delta\right.}\right)=0 .
$$

Therefore, if the HPO optimization method is no-regret with respect to the number of evaluations $B$, i.e., $R_{n}=o(1)$, the overall regret $r_{B}^{n}$ diminishes as $n$ and $B$ increase. Indeed, under some mild conditions, we can achieve $R_{n}=o(1)$ using no-regret optimization methods such as GP-UCB [35] and GP-MES [40]. Srinivas et al. [35] indicate that GP-UCB is no-regret in the sense of cumulative regret, and it is straightforward to apply the theoretical result to our notion of regret as done in $[17,18]$.

\section{EXPERIMENTS}

We investigate the behavior of our proposed HPO procedure in MSCS using a toy problem in Section 5.3 and real-world datasets in Section 5.4. The code for reproducing the results of the experiments is available at https:/github.com/nmasahiro/MS-CS.

\subsection{Baseline Methods}

We compare the following four methods as possible baselines for MS-CS: (i) Learning Initialization (LI) [41], (ii) DistBO [20], (iii): Naive method, which uses the performance on the concatenation of source tasks as a validation score, (iv) Oracle method, which uses the labeled target task for HPO. Note that the oracle method is infeasible in MS-CS, and we regard the performance of the oracle method as an upper bound to which other methods can reach.

Learning Initialization (LI) [41] aims to find promising hyperparameters by minimizing a meta-loss function. The meta-loss function is defined as the sum of a surrogated loss function on each source task. Intuitively, by minimizing the meta-loss function, LI can obtain hyperparameters that can lead to a good performance on average for source tasks.

DistBO transfers knowledge across tasks using learnt representations of training datasets [20]. To measure the similarity between these tasks, DistBO uses a distributional kernel, which learns the similarity between source and target tasks by a joint Gaussian process model. At the first iteration of optimization for the target task, DistBO uses LCB (Lower Confidence Bound) as the acquisition function to quickly select good hyperparameters. DistBO models a joint distribution $p(x, y)$ of each task. However, in MS-CS, modeling $p_{T}(x, y)$ is impossible because the labeled data of the target task are unavailable. Therefore, in our experiments, DistBO models the marginal distribution $p(x)$, not the joint distribution $p(x, y)$, of each task. This setting is also used in the original paper (Section
5.1 of [20]). If the covariate shift assumption holds, it is sufficient to model $p(x)$, as $p(y \mid x)$ does not change across tasks.

\subsection{Experimental Setting}

For fair comparison, we used Gaussian Process Upper Confidence Bound (GP-UCB) [35] as a hyperparameter optimization algorithm for all methods. In our experiments, the confidence parameter in GP-UCB is set to 2.0 (following the setting used in $[5,14,23]$ ).

We set the number of evaluations (i.e., $B$ in Algorithm 1 and 2) to 50 . At the beginning of optimization, we sample 5 initial points randomly. A Matérn 5/2 kernel is used in the implementation of GP-UCB. Note that our study is formulated as a minimization as in Eq. (1), we thus use LCB instead of UCB as an acquisition function. We use densratio_py ${ }^{3}$ to estimate the density ratio by uLSIF [16]. All the experiments were conducted on Google Cloud Platform (n1-standard-4) or MacBook Pro (2.2 GHz Intel Core i7, 16 GB).

For LI, which utilizes the gradient descent algorithm to optimize a meta-loss function defined by source tasks, we need to set a learning rate $\eta$ and a number of epochs $E$. Following [41], we set $\eta=10^{-3}$ and $E=10^{3}$. To obtain the results of DistBO in our experiments, we use the implementation provided by the authors. ${ }^{4}$ We use a Matern $5 / 2$ kernel for a fair comparison with other methods, whereas the original implementation uses a Matérn 3/2 kernel as default.

\subsection{Toy Problem}

We consider a 1-dimensional regression problem which aligns with the MS-CS setting. The toy dataset is generated as follows:

$$
\begin{gathered}
\mu^{j} \sim \mathcal{U}\left(-c_{j}, c_{j}\right), \\
\left\{x_{i}^{j}\right\}_{i=1}^{n} \mid \mu^{j} \stackrel{i . i . d .}{\sim} \mathcal{N}\left(\mu^{j}, 1\right), \\
\left\{y_{i}^{j}\right\}_{i=1}^{n} \mid\left\{x_{i}^{j}\right\}_{i=1}^{n} \stackrel{\text { i.i.d. }}{\sim}\left\{\mathcal{N}\left(0.7 x_{i}^{j}+0.3,1\right)\right\}_{i=1}^{n},
\end{gathered}
$$

where $\mathcal{U}$ is the uniform distribution, $\mathcal{N}$ denotes the normal distribution, and $c_{j} \in \mathbb{R}$ is an experimental setting which characterizes the marginal input distribution $(p(x))$ of task $j$. The objective function $f$ is given by:

$$
f\left(\theta ; \mathcal{D}_{j}\right)=\frac{1}{n} \sum_{i=1}^{n} L\left(\theta, y_{i}\right), L\left(\theta, y_{i}\right)=\left(\theta-y_{i}\right)^{2} / 2
$$

Similar to the toy experiment in [20], $\theta \in[-8,8]$ is a hypothetical hyperparameter, which we aim to optimize.

As we described in Section 3, the variance reduced estimator is expected to outperform the unbiased estimator, when the source task and the target task differ significantly. To demonstrate this, we use various values as $c_{j}$ to define source tasks $\left(c_{j}^{S} \in\{1.0,2.0, \cdots, 5.0\}, j \in\right.$ $\{1, \cdots, k\})$ and target task $\left(c^{T}=1.0\right)$. The source and target distributions are similar when $c_{j}^{S}=1.0\left(=c^{T}\right)$; in contrast, they are quite different when $c_{j}^{S}=5.0$. Finally, we set $k=2$ and $n=1000$.

Figure 2 shows the results of the experiment on the toy problem over 30 runs with different random seeds. First, Figure 2 (a) indicates that the unbiased and variance reduced estimators significantly outperform the naive method and LI in all settings. This is because these estimators unbiasedly approximate the target task objective

\footnotetext{
${ }^{3}$ https://github.com/hoxo-m/densratio_py

${ }^{4}$ https://github.com/hcllaw/distBO
} 


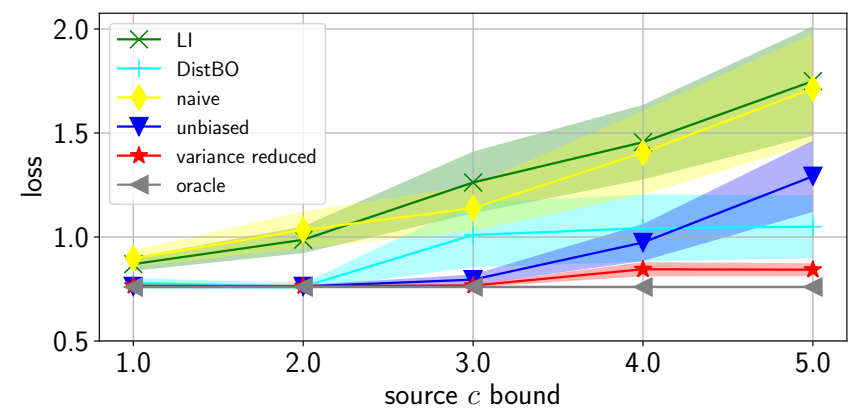

(a) Comparing all methods

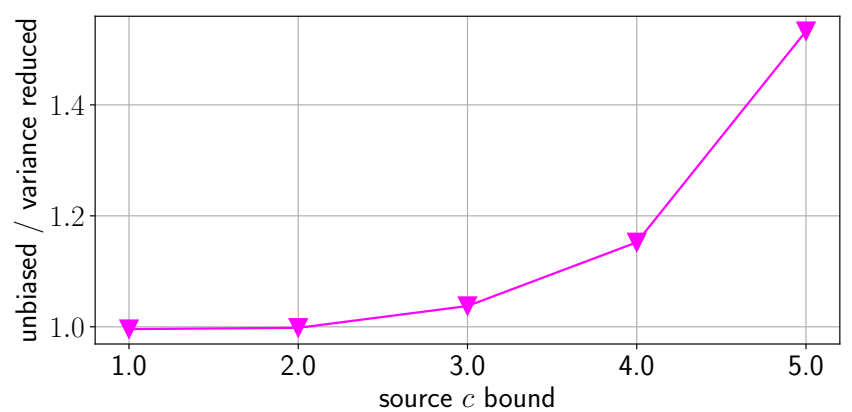

(b) Comparing unbiased and variance reduced

Figure 2: Results of the experiment on synthetic toy problems over 30 runs. The horizontal axis represents the prior parameters of the source tasks $c_{j}^{S} \in\{1.0, \cdots, 5.0\},(j \in\{1, \cdots, k\})$. (a) The vertical axis represents the mean and standard error of the performance of each method. (b) The vertical axis represents the ratio of the mean performance of the unbiased estimator to the variance reduced estimators.

by addressing the distributional shift, while the naive method and LI do not. The variance reduced estimator also outperforms DistBO when there is a source task that is greatly different from the target (when $c$ is large).

Next, we compare the performance of the unbiased and variance reduced estimators in Figure 2 (b). This reports the performance of the unbiased estimator relative to the variance reduced estimator with varying values of $c$. The result indicates that the advantage of the variance reduced estimator over the unbiased estimator is further strengthened when there is a large divergence between the target and source task distributions. This result is consistent with our theoretical analysis, which suggests that the variance reduced estimator has the minimum variance. Finally, as shown in Figure 2 (a), the variance reduced estimator achieves almost the same performance as the upper bound (oracle) without using the labels of the target task, suggesting its powerful HPO performance under the MS-CS setting.

\subsection{Real-World Data}

Datasets: We use Parkinson's telemonitoring (Parkinson) [38] and Graft-versus-host disease (GvHD) datasets [7] to evaluate our proposed method on real-world problems.

Parkinson consists of voice measurements of 42 patients with early-stage Parkinson disease collected by using a telemonitoring device in remote symptom progression monitoring. Each patient has about 150 recordings characterized by a feature vector with 17 dimensions. The goal is to predict the Parkinson disease symptom score of each recording.

GvHD is an important medical problem in the allogeneic blood transplantation field [7]. The issue occurs in allogeneic hematopoietic stem cell transplant recipients when donor immune cells in the graft recognize the recipient as foreign and initiate an attack on several tissues. The GvHD dataset contains weekly peripheral blood samples obtained from 31 patients characterized by a feature vector with 7 dimensions. Following [22], we omit one patient who has insufficient data, and subsample the data of each patient to have 1000 data points each. The goal is to classify CD3+CD4+CD8+ cells, which have a high correlation with the development of the disease [7].

Experimental Procedure: To create the MS-CS setting, we treat each patient as a task for both datasets. We select one patient as a target task and regard the remaining patients as multiple source tasks. Then, we employ the following experimental procedure:

(1) Tune hyperparameters of an ML model by an HPO method using the unlabeled target task and labeled source tasks,

(2) Split the original target task data into $70 \%$ training set and $30 \%$ test set,

(3) Train an ML model tuned by an HPO method for MS-CS using the training set of the target task,

(4) Predict target variables (the symptom score for Parkinson and CD3+CD4+CD8+ cells for GvHD) on the test set of the target patient,

(5) Calculate target task objective (prediction accuracy) and regard it as the performance of the HPO method,

(6) Repeat the above steps 10 times with different seeds and report the mean and standard error over the simulations.

Note that an HPO method can be one of LI, DistBO, Naive, Unbiased, Variance Reduced, or Oracle. An ML model is either of support vector machine (SVM) or LightGBM as described below.

Settings: As for an ML model and a target task objective, we use SVM implemented in scikit-learn [25] and mean absolute error (MAE) for Parkinson. In contrast, we use LightGBM [19] as an ML model and binary cross-entropy (BCE) as a target task objective for GvHD.

Tables 2 and 3 describe the hyperparameter search spaces $(\Theta)$ for SVM (Parkinson) and LightGBM (GvHD). We treat integervalued hyperparameters as a continuous variable and discretize them when defining an ML model. For SVM, we use Radial Basis Function kernel in scikit-learn [25]. We use microsoft/LightGBM ${ }^{5}$

$\overline{{ }^{5} \text { https://github.com/microsoft/LightGBM }}$ 
Table 2: Hyperparameter space for SVM on Parkinson's telemonitoring dataset.

\begin{tabular}{llll}
\hline Hyperparameters & Type & Scale & Search Space \\
\hline Kernel Coefficient & float & $\log$ & {$\left[5.0 \times 10^{-5}, 5.0 \times 10^{3}\right]$} \\
L2 Regularization Parameter & float & $\log$ & {$\left[5.0 \times 10^{-5}, 5.0 \times 10^{3}\right]$} \\
\hline
\end{tabular}

Table 3: Hyperparameter space for LightGBM on GvHD dataset.

\begin{tabular}{llll}
\hline Hyperparameters & Type & Scale & Search Space \\
\hline Max Depth for Tree & int & linear & {$[2,6]$} \\
Feature Fraction & float & $\operatorname{linear}$ & {$[0.1,1.0]$} \\
Learning Rate & float & $\log$ & {$\left[1.0 \times 10^{-3}, 1.0 \times 10^{-1}\right]$} \\
L2 Regularization Parameter & float & $\log$ & {$\left[5.0 \times 10^{-5}, 5.0 \times 10^{3}\right]$} \\
\hline
\end{tabular}

to implement LightGBM. For the details of these hyperparameters, please refer to the documentations of scikit-learn ${ }^{6}$ and LightGBM $^{7}$.

We normalize the feature vectors of the GvHD dataset as a preprocessing procedure. In contrast, in the Parkinson dataset, we do not apply standardization or normalization, because these operations cause serious performance degradation of SVM. In the Parkinson dataset, we select a task (patient) having the maximum number of data as the target task. In contrast, the tasks in the GvHD dataset all have the same number of data, thus we select the task (patient) with the first task index as the target. When we use unbiased and variance reduced estimators, we use $30 \%$ of the training set to estimate the density ratio and the remaining $70 \%$ to learn the ML models as suggested in Section 3.

Table 4: Comparing different MS-CS methods (Mean \pm StdErr). The red fonts represent the best performance among estimators that are feasible in MS-CS. The mean and standard error (StdErr) are induced by running 10 simulations with different random seeds.

\begin{tabular}{lcc}
\hline Estimators & Parkinson (MAE) & GvHD (BCE) \\
\hline \hline LI & $0.41507 \pm 0.1669$ & $0.19695 \pm 0.0468$ \\
DistBO & $1.54202 \pm 0.1006$ & $0.33015 \pm 0.0600$ \\
Naive & $1.10334 \pm 0.0908$ & $0.02121 \pm 0.0052$ \\
Unbiased & $1.08283 \pm 0.1981$ & $0.02141 \pm 0.0052$ \\
Variance reduced (ours) & $\mathbf{0 . 4 0 4 5 5} \pm \mathbf{0 . 1 7 5 5}$ & $\mathbf{0 . 0 1 7 9 1 \pm 0 . 0 0 3 9}$ \\
\hline Oracle (reference) & $0.06862 \pm 0.0011$ & $0.01584 \pm 0.0043$ \\
\hline
\end{tabular}

Results: Table 4 presents the results of the experiments over 10 runs with different random seeds. In contrast to the results on synthetic data, the performance of DistBO deteriorates significantly. While DistBO requires a reasonable number of hyperparameter evaluations per source task, our setting allows only a very small number of evaluations per source task, which may lead to learning inaccurate surrogate models for DistBO. The unbiased estimator performs almost the same with naive on the Parkinson dataset given

\footnotetext{
${ }^{6} \mathrm{https} / / /$ scikit-learn.org/stable/modules/generated/sklearn.svm.SVR.html

${ }^{7}$ https://lightgbm.readthedocs.io/en/latest/Parameters.html
}

their standard errors. Moreover, it slightly underperforms the naive in GvHD, although the unbiased estimator satisfies unbiasedness. This is because the number of data for each task is small, and the variance issue of the unbiased estimator is highlighted in these data. This result implies that pursuing only unbiasedness in the approximation of the target task objective is not sufficient in MS-CS. On the other hand, the proposed variance reduced estimator alleviates the instability issue of the unbiased estimator and performs the best in both datasets. The results also suggest that the variance reduced estimator works well on both regression (Parkinson) and classification (GvHD) problems. Therefore, we conclude from its variance optimality and empirical performance that the variance reduced estimator is the best choice to conduct efficient HPO under MS-CS.

\section{CONCLUSION}

In this work, we explore a novel HPO problem under MS-CS with the goal of enabling efficient HPO with only an unlabeled target task and multiple labeled source task datasets. Towards that end, we propose the variance reduced estimator and show that it achieves the variance optimality by leveraging the task divergence measure. Moreover, we show that the resulting HPO procedure is no-regret even under the difficult MS-CS setting. Empirical evaluation demonstrated that the proposed HPO procedure helps us identify useful hyperparameters without the labels of the target task.

In assumption 2, we assumed that the conditional outcome distribution remains the same across all tasks. Although it is a standard assumption in the covariate shift literature, it might not hold in uncertain real-world scenarios. Exploring the effective HPO procedure without this assumption, for example, by leveraging a few labeled samples from the target task, is an interesting future research direction. Another limitation of our study is that the scales of the HPO experiments are small. This is due to the fact that there are limited real-world datasets that enable the experiments under MS-CS. We argue that empirically verifying our proposed HPO procedure on larger datasets is necessary to make our procedure more convincing and reliable. 


\section{REFERENCES}

[1] Aman Agarwal, Soumya Basu, Tobias Schnabel, and Thorsten Joachims. 2017. Effective evaluation using logged bandit feedback from multiple loggers. In Proceedings of the 23rd ACM SIGKDD International Conference on Knowledge Discovery and Data Mining. 687-696.

[2] Rémi Bardenet, Mátyás Brendel, Balázs Kégl, and Michele Sebag. 2013. Collaborative hyperparameter tuning. In International conference on machine learning. 199-207.

[3] James Bergstra and Yoshua Bengio. 2012. Random search for hyper-parameter optimization. Fournal of machine learning research 13, Feb (2012), 281-305.

[4] James S Bergstra, Rémi Bardenet, Yoshua Bengio, and Balázs Kégl. 2011. Algorithms for Hyper-Parameter Optimization. In Advances in neural information processing systems. 2546-2554.

[5] Ilija Bogunovic, Jonathan Scarlett, Stefanie Jegelka, and Volkan Cevher. 2018. Adversarially robust optimization with gaussian processes. In Advances in neural information processing systems. $5760-5770$.

[6] Edwin V Bonilla, Kian M Chai, and Christopher Williams. 2008. Multi-task Gaussian Process Prediction. In Advances in neural information processing systems. 153-160.

[7] Ryan Remy Brinkman, Maura Gasparetto, Shang-Jung Jessica Lee, Albert J Ribickas, Janelle Perkins, William Janssen, Renee Smiley, and Clay Smith. 2007 High-content flow cytometry and temporal data analysis for defining a cellular signature of graft-versus-host disease. Biology of Blood and Marrow Transplantation 13, 6 (2007), 691-700.

[8] Eric Brochu, Vlad M Cora, and Nando De Freitas. 2010. A tutorial on Bayesian optimization of expensive cost functions, with application to active user modeling and hierarchical reinforcement learning. arXiv preprint arXiv:1012.2599 (2010).

[9] Maurizio Ferrari Dacrema, Paolo Cremonesi, and Dietmar Jannach. 2019. Are We Really Making Much Progress? A Worrying Analysis of Recent Neural Recommendation Approaches. In Proceedings of the 13th ACM Conference on Recommender Systems. 101-109.

[10] Víctor Elvira, Luca Martino, David Luengo, and Mónica F Bugallo. 2015. Efficient multiple importance sampling estimators. IEEE Signal Processing Letters 22, 10 (2015), 1757-1761.

[11] Matthias Feurer and Frank Hutter. 2019. Hyperparameter Optimization. In Automated Machine Learning. 3-33.

[12] Matthias Feurer, Benjamin Letham, and Eytan Bakshy. 2018. Scalable MetaLearning for Bayesian Optimization using Ranking-Weighted Gaussian Process Ensembles. In AutoML Workshop at ICML.

[13] Peter I Frazier. 2018. A tutorial on bayesian optimization. arXiv preprint arXiv:1807.02811 (2018).

[14] Javier González, Zhenwen Dai, Philipp Hennig, and Neil Lawrence. 2016. Batch bayesian optimization via local penalization. In Artificial intelligence and statistics. 648-657.

[15] Peter Henderson, Riashat Islam, Philip Bachman, Joelle Pineau, Doina Precup, and David Meger. 2018. Deep Reinforcement Learning that Matters. In Thirty-Second AAAI Conference on Artificial Intelligence.

[16] Takafumi Kanamori, Shohei Hido, and Masashi Sugiyama. 2009. A least-squares approach to direct importance estimation. Journal of Machine Learning Research 10, Jul (2009), 1391-1445.

[17] Kirthevasan Kandasamy, Gautam Dasarathy, Junier Oliva, Jeff Schneider, and Barnabas Poczos. 2019. Multi-fidelity gaussian process bandit optimisation Fournal of Artificial Intelligence Research 66 (2019), 151-196.

[18] Kirthevasan Kandasamy, Akshay Krishnamurthy, Jeff Schneider, and Barnabás Póczos. 2018. Parallelised bayesian optimisation via thompson sampling. In International Conference on Artificial Intelligence and Statistics. PMLR, 133-142.

[19] Guolin Ke, Qi Meng, Thomas Finley, Taifeng Wang, Wei Chen, Weidong Ma, Qiwei Ye, and Tie-Yan Liu. 2017. Lightgbm: A highly efficient gradient boosting decision tree. In Advances in neural information processing systems. 3146-3154.

[20] Ho Chung Law, Peilin Zhao, Leung Sing Chan, Junzhou Huang, and Dino Sejdinovic. 2019. Hyperparameter learning via distributional transfer. In Advances in Neural Information Processing Systems. 6801-6812.

[21] Mario Lucic, Karol Kurach, Marcin Michalski, Sylvain Gelly, and Olivier Bousquet. 2018. Are Gans Created Equal? A Large-Scale Study. In Advances in neural information processing systems. 700-709.

[22] Krikamol Muandet, David Balduzzi, and Bernhard Schölkopf. 2013. Domain generalization via invariant feature representation. In International Conference on Machine Learning. 10-18.

[23] Vu Nguyen, Santu Rana, Sunil K Gupta, Cheng Li, and Svetha Venkatesh. 2016 Budgeted batch Bayesian optimization. In 2016 IEEE 16th International Conference on Data Mining (ICDM). IEEE, 1107-1112.

[24] Masahiro Nomura, Shuhei Watanabe, Youhei Akimoto, Yoshihiko Ozaki, and Masaki Onishi. 2021. Warm Starting CMA-ES for Hyperparameter Optimization. In Proceedings of the AAAI Conference on Artificial Intelligence, Vol. 35. 9188-9196.

[25] Fabian Pedregosa, Gaël Varoquaux, Alexandre Gramfort, Vincent Michel, Bertrand Thirion, Olivier Grisel, Mathieu Blondel, Peter Prettenhofer, Ron Weiss, Vincent Dubourg, et al. 2011. Scikit-learn: Machine learning in Python. the
Journal of machine Learning research 12 (2011), 2825-2830.

[26] Valerio Perrone, Rodolphe Jenatton, Matthias W Seeger, and Cédric Archambeau. 2018. Scalable Hyperparameter Transfer Learning. In Advances in Neural Information Processing Systems. 6845-6855.

[27] Valerio Perrone, Huibin Shen, Matthias W Seeger, Cedric Archambeau, and Rodolphe Jenatton. 2019. Learning search spaces for Bayesian optimization: Another view of hyperparameter transfer learning. In Advances in Neural Information Processing Systems. 12751-12761.

[28] Matthias Poloczek, Jialei Wang, and Peter Frazier. 2017. Multi-information source optimization. In Advances in Neural Information Processing Systems. 4288-4298.

[29] Anil Ramachandran, Sunil Gupta, Santu Rana, and Svetha Venkatesh. 2018. Information-theoretic Transfer Learning framework for Bayesian Optimisation. In foint European Conference on Machine Learning and Knowledge Discovery in Databases. 827-842.

[30] David Salinas, Huibin Shen, and Valerio Perrone. 2020. A quantile-based approach for hyperparameter transfer learning. In International Conference on Machine Learning. PMLR, 8438-8448.

[31] Bobak Shahriari, Kevin Swersky, Ziyu Wang, Ryan P Adams, and Nando De Freitas. 2015. Taking the human out of the loop: A review of Bayesian optimization. Proc. IEEE 104, 1 (2015), 148-175.

[32] Hidetoshi Shimodaira. 2000. Improving predictive inference under covariate shift by weighting the log-likelihood function. fournal of statistical planning and inference 90, 2 (2000), 227-244

[33] Jasper Snoek, Hugo Larochelle, and Ryan P Adams. 2012. Practical bayesian optimization of machine learning algorithms. In Advances in neural information processing systems. 2951-2959.

[34] Jost Tobias Springenberg, Aaron Klein, Stefan Falkner, and Frank Hutter. 2016. Bayesian Optimization with Robust Bayesian Neural Networks. In Advances in Neural Information Processing Systems. 4134-4142.

[35] Niranjan Srinivas, Andreas Krause, Sham Kakade, and Matthias Seeger. 2010. Gaussian process optimization in the bandit setting: no regret and experimental design. In Proceedings of the 27th International Conference on International Conference on Machine Learning. 1015-1022.

[36] Masashi Sugiyama, Matthias Krauledat, and Klaus-Robert MÃžller. 2007. Covariate shift adaptation by importance weighted cross validation. Fournal of Machine Learning Research 8, May (2007), 985-1005.

[37] Kevin Swersky, Jasper Snoek, and Ryan P Adams. 2013. Multi-Task Bayesian Optimization. In Advances in neural information processing systems. 2004-2012.

[38] Athanasios Tsanas, Max A Little, Patrick E McSharry, and Lorraine O Ramig. 2009. Accurate telemonitoring of Parkinson's disease progression by noninvasive speech tests. IEEE transactions on Biomedical Engineering 57, 4 (2009), 884-893.

[39] Ryan Turner, David Eriksson, Michael McCourt, Juha Kiili, Eero Laaksonen, Zhen $\mathrm{Xu}$, and Isabelle Guyon. 2021. Bayesian optimization is superior to random search for machine learning hyperparameter tuning: Analysis of the black-box optimization challenge 2020. arXiv preprint arXiv:2104.10201 (2021).

[40] Zi Wang and Stefanie Jegelka. 2017. Max-value entropy search for efficient Bayesian optimization. In International Conference on Machine Learning. PMLR, 3627-3635.

[41] Martin Wistuba, Nicolas Schilling, and Lars Schmidt-Thieme. 2015. Learning hyperparameter optimization initializations. In 2015 IEEE international conference on data science and advanced analytics (DSAA). IEEE, 1-10.

[42] Martin Wistuba, Nicolas Schilling, and Lars Schmidt-Thieme. 2018. Scalable gaussian process-based transfer surrogates for hyperparameter optimization. Machine Learning 107, 1 (2018), 43-78.

[43] Makoto Yamada, Taiji Suzuki, Takafumi Kanamori, Hirotaka Hachiya, and Masashi Sugiyama. 2011. Relative density-ratio estimation for robust distribution comparison. In Advances in neural information processing systems. 594-602.

[44] Dani Yogatama and Gideon Mann. 2014. Efficient transfer learning method for automatic hyperparameter tuning. In Artificial intelligence and statistics. 10771085.

[45] Kaichao You, Ximei Wang, Mingsheng Long, and Michael Jordan. 2019. Towards accurate model selection in deep unsupervised domain adaptation. In International Conference on Machine Learning. 7124-7133.

[46] Erheng Zhong, Wei Fan, Qiang Yang, Olivier Verscheure, and Jiangtao Ren. 2010. Cross validation framework to choose amongst models and datasets for transfer learning. In foint European Conference on Machine Learning and Knowledge Discovery in Databases. Springer, 547-562. 